\title{
Fourier transform spectral interferometry with non-phase stable setups by broadband single shot detection of fs and ps pulses
}

Georg Achazi, and Andrea Cannizzo

Citation: Review of Scientific Instruments 88, 083110 (2017); doi: 10.1063/1.4998989

View online: https://doi.org/10.1063/1.4998989

View Table of Contents: http://aip.scitation.org/toc/rsi/88/8

Published by the American Institute of Physics

\section{Articles you may be interested in}

Coherent multi-dimensional spectroscopy at optical frequencies in a single beam with optical readout The Journal of Chemical Physics 147, 094203 (2017); 10.1063/1.4990500

Instrumentation for ice crystal characterization in laboratory using interferometric out-of-focus imaging Review of Scientific Instruments 88, 083108 (2017); 10.1063/1.4997959

Sine wave gating silicon single-photon detectors for multiphoton entanglement experiments

Review of Scientific Instruments 88, 083102 (2017); 10.1063/1.4986038

Development of a $10 \mathrm{kHz}$ high harmonic source up to $140 \mathrm{eV}$ photon energy for ultrafast time-, angle-, and phase-resolved photoelectron emission spectroscopy on solid targets

Review of Scientific Instruments 88, 083105 (2017); 10.1063/1.4989399

Signatures of vibronic coupling in two-dimensional electronic-vibrational and vibrational-electronic spectroscopies

The Journal of Chemical Physics 147, 094202 (2017); 10.1063/1.4991745

Micrometer-thickness liquid sheet jets flowing in vacuum

Review of Scientific Instruments 88, 083117 (2017); 10.1063/1.4990130

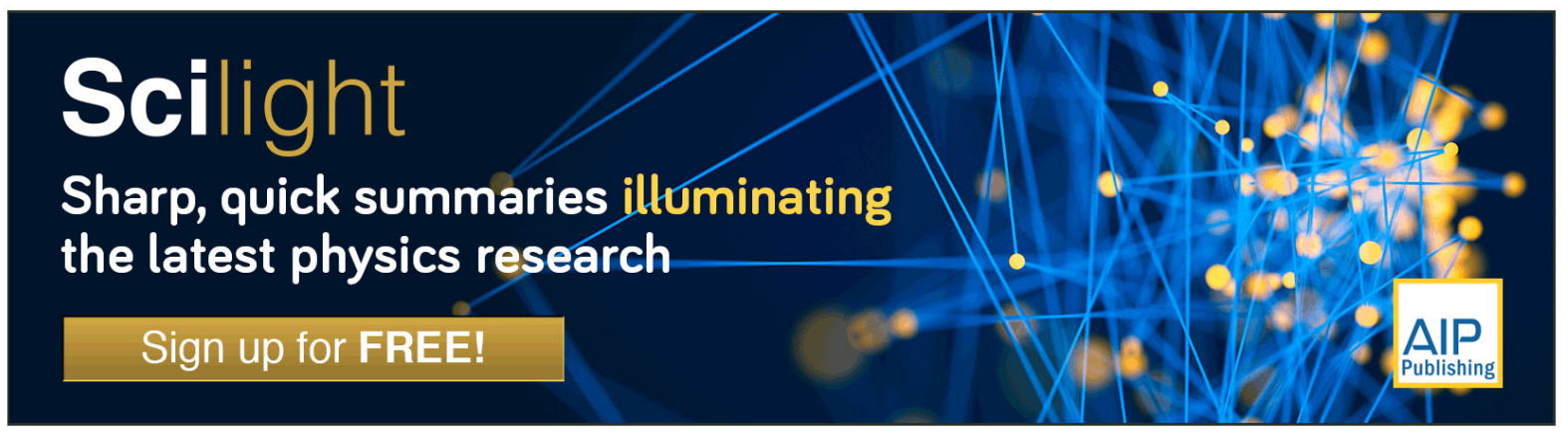




\title{
Fourier transform spectral interferometry with non-phase stable setups by broadband single shot detection of fs and ps pulses
}

\author{
Georg Achazi and Andrea Cannizzo a) \\ Institute of Applied Physics, University of Bern, Sidlerstrasse 5, CH-3012 Bern, Switzerland
}

(Received 8 May 2017; accepted 1 August 2017; published online 18 August 2017)

\begin{abstract}
In this paper, we present a novel approach to Fourier transform spectral interferometry based on singleshot detection of broadband pulses. It allows heterodyne detection with non-phase-stabilised setups, which use ps- and fs-pulsed laser sources. This approach can tremendously simplify the implementation of complex techniques such as heterodyne Fourier transform four-wave mixing and pave the way to novel phase-sensitive diagnostics for lasers and optical setups. Published by AIP Publishing. [http://dx.doi.org/10.1063/1.4998989]
\end{abstract}

\section{INTRODUCTION}

In the last two decades, there has been an impulse to develop interferometric schemes with fs pulsed laser sources after the successful implementation of the first Fourier transform (FT) four-wave mixing (FWM) experiments. The most common methods are photon echo spectroscopy, coherent anti-stokes Raman spectroscopy, and transient grating spectroscopy. ${ }^{1-6}$ In these experiments, the nonlinear response of the sample is measured by detecting the amplitude and phase of an electromagnetic field generated by the sample (signal), in contrast to conventional techniques where only the power spectrum is recorded. This is achieved by means of optical heterodyne detection, which is ultimately the detection of the interference between the signal and an external known field, the so-called local oscillator. ${ }^{5}$ These methodologies have proven to provide great insight and represent the state of the art modern optical spectroscopy.

In such an experiment, several beams interact with the sample and any change in the relative phase will be transferred to the signal phase. ${ }^{1}$ When the extent of such fluctuations is comparable to the wavelength of the signal, the interference pattern is dramatically degraded. The main source of phase fluctuations is mechanical vibrations, air turbulences, and thermal dilatation, which randomly change the optical path of the different beams. Conventionally a phase-stable setup should provide a phase-jittering less than $\frac{\lambda}{100}$, where $\lambda$ is the central wavelength of the signal. This condition is ideal for a FT analysis but poorer stabilities could be acceptable depending on the desired phase fidelity. Of course this value is not an absolute feature of the setup since it depends on the wavelength and because fluctuations have different characteristic times. Indeed the detector integration time will discriminate between phase noise (phase fluctuations that occur within the integration time) and phase drift (phase fluctuations slow enough to be irrelevant during the acquisition). Achieving the required phase stability is the most challenging task when constructing a FT-FWM setup.

a)andrea.cannizzo@iap.unibe.ch
The most common scheme uses two pairs of phase stable pulses in a non-collinear boxcar configuration. The first pair is the two pump pulses while the second pair consists of the probe pulse and the local oscillator. In the IR, phase noise can usually be suppressed by using highly stable mounts for all optics ${ }^{5}$ and phase shift by active phase stabilization. ${ }^{7}$ For FT-FWM electronic spectroscopy with visible and UV pulses, an inherently phase stable setup is needed. ${ }^{6}$ A milestone was the implementation of passive phase stabilized setups based on the reflection of the conjugated beams by the same optical elements. ${ }^{8-10}$ In an alternate geometry, a pair of phase stable collinear pump pulses is created with a one-dimensional pulse shaper, ${ }^{11,12}$ while the non-collinear probe pulse is also used as a local oscillator. This allows us to improve the signal-to-noise ratio by phase cycling and to control the inter-pulse delay between the pump pulses with attosecond precision without using mechanical parts. ${ }^{13}$ However, it sacrifices the ability to adapt delay and intensity of the local oscillator to optimize the signal and limits to few ps the range of variability for the delays between pulses. ${ }^{13,14}$ More advanced setups combine the benefits of boxcar geometry and pulse shaping by using a phase mask in combination with a two-dimensional pulse shaper, to create four phase stable pulses where the spectral amplitude, the phase, and the interpulse delay of each pulse can be precisely and individually controlled. ${ }^{13,14}$

The current efforts in the field of FT-FWM are aimed at extending these techniques to shorter spectral wavelengths to access aromatic amino acids and DNA nucleotide bases ${ }^{15}$ and wide gap materials like $\mathrm{TiO}_{2}$ or even core transitions. ${ }^{16}$ These experiments are now conceivable because of the development of new sources for broadband deep-UV-pulses, ${ }^{17,18}$ EUV by high harmonics generation, ${ }^{19}$ and soft and hard X-ray by free-electron-lasers. ${ }^{16}$ However due to shorter and shorter wavelengths, phase stability is more and more an issue. The strategy adopted so far has been to improve the optical path stability, but this is not always possible and cannot be indefinitely pushed forward.

Here we present a new approach based on the single shot detection of fs-pulses, which can make phase stabilization unnecessary and present schemes obsolete. 


\section{THEORY}

All the main applications of spectral interferometry rely on its capability to reconstruct electromagnetic fields (namely, amplitude and phase). One of the most notable examples is optical heterodyne detection, which is used in FT spectroscopy to measure signals generated by the sample by detecting the interference between the signal and the local oscillator. The spectral intensity $I(\omega)$ of the measured interferogram can be described by the following formula: ${ }^{20}$

$$
\begin{aligned}
I(\omega)= & \left|E(\omega)_{s}\right|^{2}+\left|E(\omega)_{l o}\right|^{2} \\
& +2\left|E(\omega)_{s}\right|\left|E(\omega)_{l o}\right| \cos (\Delta \varphi(\omega)) .
\end{aligned}
$$

$E(\omega)_{s}$ and $E(\omega)_{l o}$ represent the electric field of the signal and the local oscillator, respectively, and $\Delta \varphi(\omega)=\varphi(\omega)_{s}$ $-\varphi(\omega)_{l o}+\omega \cdot(\tau+\delta)$ is the difference between the phases of the fields $\varphi(\omega)_{s}$ and $\varphi(\omega)_{l o}$, plus the delay contribution $\omega \cdot(\tau+\delta)$, where $\tau$ is the inter-pulse delay and $\delta$ is an instantaneous optical path fluctuation. With this notation, the presence of phase fluctuations without phase drift would imply that the temporal average over the integration time of $\delta(\langle\delta\rangle)$ is 0 . Conversely in the case of a slow phase-shift, $\delta$ does not fluctuate around zero but drifts. From the interferogram of Eq. (1), the complex field

$$
\widehat{S}(\omega)=\left|E(\omega)_{s}\right|\left|E(\omega)_{l o}\right| e^{i\left(\varphi(\omega)_{s}-\varphi(\omega)_{l o}+\omega \cdot(\tau+\delta)\right)},
$$

can be extracted and the electric field of the signal can be obtained by dividing $\widehat{S}(\omega)$ by the field of the local oscillator. Figure 1 shows the steps of this method for 4 interferograms with different pulse delays $\tau$.

The main criticality of such detection is that phase instabilities irreversibly degrade or even destroy an interferogram upon averaging [see Eq. (1) and Fig. 1(a)].

The main source of phase noise is usually mechanical instabilities in the setup due to vibrations and drifts of reflective surfaces during the integration time, which change the delay between the signal and local oscillator. In a non-compact setup, the effects of air turbulences could also be non-negligible. However, when pulsed light sources are used, the integration time should be carefully defined taking into account also the length of the pulse. Indeed when a short pulse light source is used to generate interferograms with a broadband single-shot detection, the effective integration time is given by the pulse length and not by the detector electronics. Accordingly, in the case of fs and ps pulses, phase noise due to mechanical instabilities can be neglected on a Single Shot detected Interferogram (SSI): for instance, considering a pulse length of $100 \mathrm{fs}$ and as a maximal acceptable fluctuation of the optical pathway $\Delta \lambda \leq 1$ $\mathrm{nm}$ (i.e., the optimal condition for $\lambda=100 \mathrm{~nm}$ ) mirror surfaces should move with a speed of $\frac{1 \mathrm{~nm}}{100 \mathrm{fs}}=10 \frac{\mathrm{km}}{\mathrm{s}}$ to induce a phasefluctuation able to perturb a SSI. This is 30 times the speed of sound in air and will never be achieved. Actually taking as a more reasonable order of magnitude $1 \mathrm{~mm} / \mathrm{s}$ (which is a coarse and very conservative estimation depending on the total number of reflective surfaces in the setup, their substrate thickness, the quality of the mounts and the overall optical design, the environmental vibrational standard), the criterion of $\Delta \lambda=\frac{\lambda}{100}$ should hold until $\lambda=10^{-3} \AA$ (or $10^{4} \mathrm{keV}$ ) with 1 ps pulses.
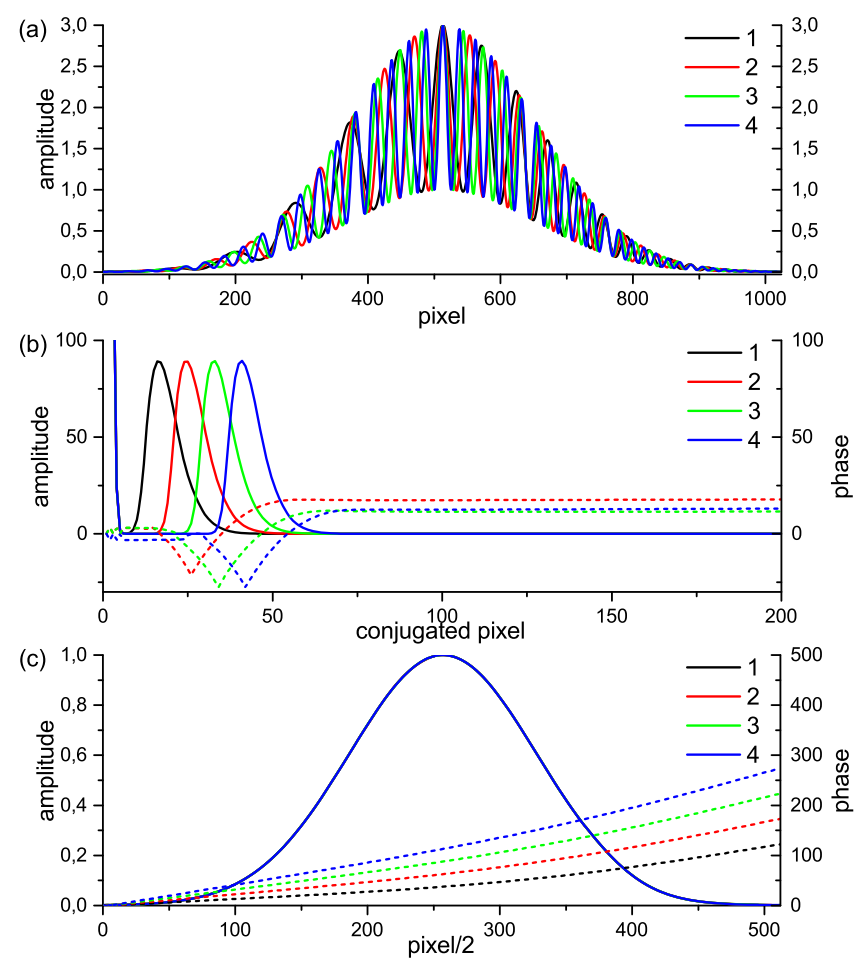

FIG. 1. Detection and analysis of heterodyne signals. For the sake of clarity, the method is illustrated on simulated signals at different delays between the signal and local oscillator, both assumed with the same power spectrum. To extract the field of the signal [Eq. (2)], the interferogram (a) described by Eq. (1) is first transformed by an inverse Fourier transformation into complex space (b). Then a windowing function (a super-Gaussian was chosen) around the interference peak is applied to reduce the noise and cut out the zerofrequency-terms [the first two terms in Eq. (1)]. Finally this is transformed back with a complex Fourier transformation (c). These complex fields differ only by a linear contribution to the phase function. In (b) and (c), solid and dashed lines refer to amplitude (left axis) and phases (right axis), respectively. Depending on the used software packages, the first and the second steps could be inverted (namely, FT first and inverse FT later).

However at so short wavelengths, the assumption that random fluctuations of optical paths are the main source of noise could no longer be valid, as other sources of phase instabilities (as density differences of air, vibrations at the mirror surface, and surface imperfections) could become relevant. This important point will be discussed more in details at the end of the section titled Results and discussion, where we will show that the proposed approach should be still valid for $\lambda>1 \AA(<10 \mathrm{keV})$, and shorter values are still accessible with special care.

The simple calculation above proves that a SSI should be perfectly preserved also with conventional, not phasestabilized setups if fs to ps pulses are used. Therefore, it should always be possible to measure an interferogram with single shot detection and the fringes should be lost only if several shots are averaged without special care. Remarkably, this consideration should be valid for any of the currently available fs and ps sources from IR to hard-X-rays.

A less immediate but innovative advantage is the possibility to transform shot-by-shot each SSI from real space of the detector into complex space upon FT as described in Fig. 1 and averaging amplitude and phase separately [Eq. (2) and Fig. 1(c)] instead of the detected real signals [Eq. (1) and Fig. 1(a)]. The consequence of switching from averaging 
real quantities to complex ones is dramatic: from Eqs. (1) and (2) and assuming for the sake of exemplification $\langle\delta\rangle=0$ and $\omega \sigma_{\delta} \gg 2 \pi$ (where $\sigma_{\delta}$ is the standard deviation of the $\delta$ distribution), we can easily derive that an averaged real signal $\langle I(\omega)\rangle$ is proportional to $\langle\cos (\Delta \varphi(\omega))\rangle=0$ (neglecting the zero-frequency terms), while the angular phase $\langle\Delta \varphi(\omega)\rangle$ $=\left\langle\varphi_{S}(\omega)-\varphi_{L O}(\omega)+\omega \tau+\omega \delta\right\rangle=\varphi_{S}(\omega)-\varphi_{L O}(\omega)+\omega \tau$ will benefit from averaging. (The same trivially holds for averaging the amplitude $\left\langle\left|E(\omega)_{s}\right|\left|E(\omega)_{l o}\right|\right\rangle$.) This approach allows also measuring shot-by-shot the delay fluctuations $\delta$. Therefore slow drifts and fast fluctuations can be precisely characterized and compensated, considerably reducing the required number of acquired spectra.

\section{EXPERIMENTAL SETUP AND DATA ANALYSIS}

To test this concept, our strategy was to build a phasestable Michelson interferometer and generate phase instability by different means. In this way, we can compare reconstructed spectra with conventional ones. Broadband visible pulses with a central wavelength of $620 \mathrm{~nm}$ and a FWHM of $50 \mathrm{~nm}$ were generated by a commercial non-collinear optical parametric amplifier (TOPAS-white, Light Conversion LTD) pumped with a $5 \mathrm{kHz}$ Legend Elite Ti:Sa laser system (CoHERENT). The pulse length was not Fourier limited but with a typical value of 150-200 fs. After the interferometer, the signal is dispersed by a transmissive grating with 830 grooves $/ \mathrm{mm}$ and focused by a $25 \mathrm{~cm}$ focal lens on a single shot $\mathrm{kHz}$ camera developed by our group in cooperation with Synertronic Designs (model Glaz PulseSync).

The camera has a CMOS detector with 1024 pixels and spectral range from $200 \mathrm{~nm}$ to $1000 \mathrm{~nm}$. It was developed to achieve single shot detection with a signal-to-noise ratio of $10^{3}$, a scan rate of up to $8.5 \mathrm{kHz}$, an integration time as short as $2 \mu \mathrm{s}$, and sensitivity down to $\mathrm{fJ} /$ pixel pulse energies.

Noise was induced in two ways: (1) by tapping on a delay stage in the interferometer to simulate mechanical instabilities and (2) by blowing pressurized $\mathrm{CO}_{2}$ in the setup to simulate air turbulences.

First we collected data in optimal illumination conditions to prove the concept, then we explored the capability to retrieve complex fields in more realistic conditions by carrying out measurements with (1) the signal-to-noise ratio $<1$ (low power limit) and (2) inserting dispersive materials into one arm of the interferometer to prove the capabilities to retrieve phase dispersion and to operate with ps pulses, provided to have enough spectral resolution. If not specified otherwise scans of 5000 SSIs each were measured.

For the conventional analysis (CA), the measured SSIs were averaged in the detection space [namely, $I(\omega)$ from Eq. (1)] and analysed as described in Sec. II. The proposed Single Shot Analysis (SSA) method was performed by calculating shot-by-shot $\widehat{S}(\omega)$ for each SSI and averaging amplitudes and phases separately. In both cases, the chirp parameters $\left(c_{n}\right)$ of the phase dispersion curves were retrieved by fitting them with a polynomial around the central wavelength,

$$
\Delta \varphi(\omega)=\sum \frac{c_{n}}{n !}\left(\omega-\omega_{0}\right)^{n} .
$$

The first 4 coefficients ( $n=0-3$ ) were enough to properly fit the experimental phase curves. In the following, we will also use the alternative notation of $\tau$ (inter-pulse delay), GVD (group velocity dispersion) and TOD (third order dispersion) for $c_{1}, c_{2}$, and $c_{3}$, respectively. The coefficient retrieval was done on the averaged phase but also on the phase of each single shot separately. This allows us to also carry analyses impossible with conventional methods as, for instance, retrieving statistical distribution and errors $\left(\sigma_{c_{n}}^{S S A}\right)$ for each chirp coefficient (which report on the quality of the setup phase stability) and characterising sources of noise and drifts of the signal. Other SSA methods like compensating and averaging in the complex conjugated space after the first Fourier transformation were tested but ultimately discarded. In fact, these methods rely on a precise shifting of the spectra for a perfect overlap of the sharp features of the phase functions [compare the dashed lines in Fig. 1(b)], which required additional mathematical manipulation like unwrapping and interpolation procedures. These manipulations add small computational and rounding errors to the phase uncertainty, which increases by $\sqrt{N}$ with the number of averaged scans $N$. As soon as the critical value of $2 \pi$ is approached, the signal is washed out.

We found that averaging fields after the second Fourier transformation is a smarter approach. It avoids the aforementioned problem because, in this space, phase-dispersion, curves are usually smoother and it is not necessary to compensate for any shift [compare Fig. 1(c)]. To further reduce the amount of mathematical manipulations, the interpolation from the detector pixel space into frequencies was done at the very end on the final averaged data, after the second Fourier transformation and the averaging. That is why some figures show data in pixel and conjugated pixel spaces, instead of the usual frequency and time spaces.

\section{RESULTS AND DISCUSSION}

To validate the proposed SSA method, we collected scans without and with phase noise. The former was conventionally analysed (method CA) to provide a benchmark reference to the SSA of the latter. Since we regularly observed much bigger phase noise upon mechanical perturbation than upon gas blowing, we will discuss in the following mainly the former. An example for the effect of air turbulences will be present at the end. The measurements were collected with a pulse energy of roughly $0.5 \mathrm{~nJ}$ (ca. $500 \mathrm{fJ} /$ pixel at maximum), corresponding to a signal close to $1 / 5$ of the camera dynamical range.

Figure 2 shows the effect of noise in the case of conventional averaging. The clear interference pattern of the unperturbed scan (red line) is destroyed by vibrational fluctuations of the setup (green line). Figure 2 shows also a representative SSI from the dataset with phase noise (black line), which definitively proves our expectation: a single-shot detected interferogram generated by a fs pulse is completely unaffected by mechanical instabilities and is substantially the same as the unperturbed ones conventionally analysed.

To validate the SSA, both the perturbed and the unperturbed dataset were analysed using this method. The results are shown in Fig. 3 (the SSA of the unperturbed data is not plotted since it is indistinguishable from the CA one) and the phase 


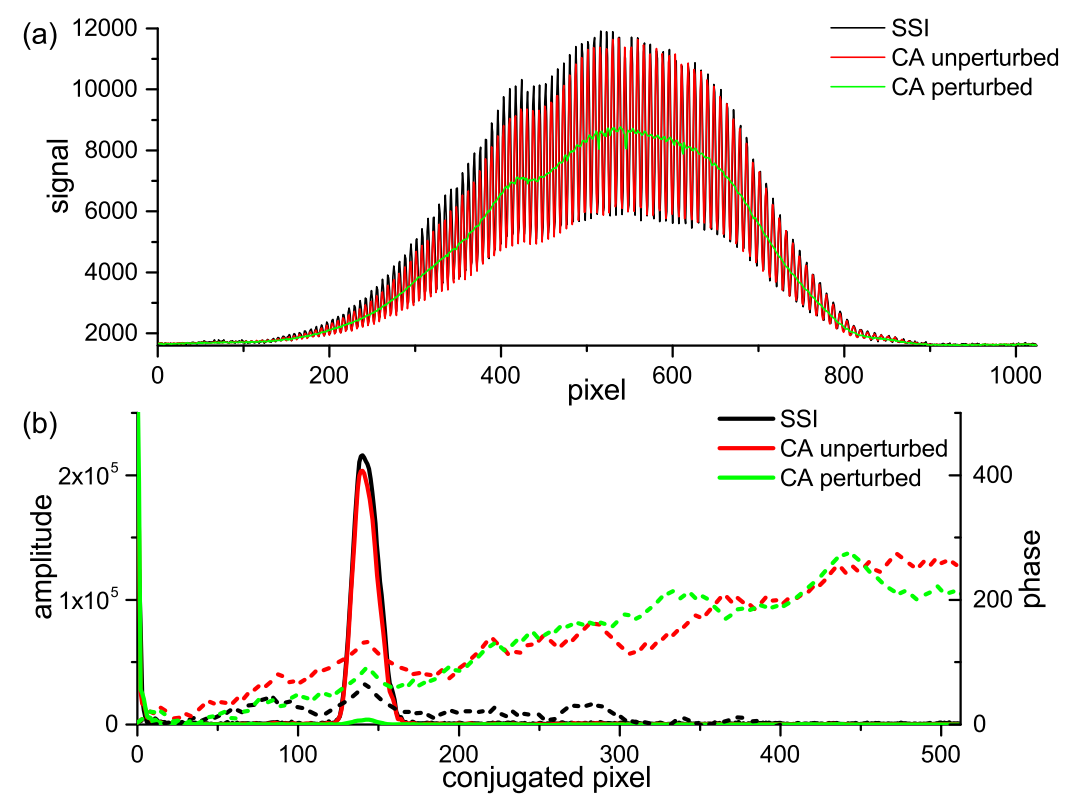

FIG. 2. Comparison between one single shot interferogram (SSI, black line) and the conventional analysis (CA) of 5000 shots measured with (CA perturbed, green line) and without (CA unperturbed, red line) mechanical fluctuations. Data are shown (a) in the detector pixel space and (b) in the conjugated pixel space after the Fourier transformation (see Fig. 1 for more details). Solid and dashed lines in (b) show magnitude and phase, respectively. parameters are given in Table I. The excellent match between SSA and CA of the same unperturbed dataset proves that in the case of a phase-stable setup, the two methods are equivalent and that the SSA does not cause any artefact or error. The comparison between the perturbed and the unperturbed dataset is overall very good but still a small linear deviation in the differential phase is observed. To clarify if it is an artefact due to the SSA or it is a real shift, we analysed the time dependence of $\tau$ of the first 1000 SSIs (Fig. 4).

While $\tau$ in the case of unperturbed data (black line) is constant with an rms deviation of 120 as, the one perturbed by tapping (red line) shows big fluctuations with a damped oscillatory pattern. Remarkably, we can clearly identify the moment when the perturbation is caused. We observe that the red line is not oscillating around the black line and the average $\tau$ of the perturbed dataset is bigger than the one of the unperturbed scan. This accounts for the linear residual phase in
Fig. 3 and the $\tau$ values in Table I. This asymmetry very likely originates from the construction design of the manual delay stage where the perturbation is applied, which has a $\mu \mathrm{m}$-screw on one side of the slide and a spring on the other. In addition, a small offset was caused by tapping as revealed by the fact that the damped oscillations seem not to converge back to the black line. This speaks for a small change in the average position of the stage upon tapping. When comparing the SSA of the perturbed and unperturbed scans, it is remarkable that while the standard deviation of $\tau$ of the perturbed scan is 60 times bigger than the one of the unperturbed scan, the standard deviations of GVD and TOD are almost identical. Another remarkable feature is that the SSI shows an uncertainty of $\tau$ of 20 as $(\Delta \lambda=6 \mathrm{~nm})$, which is the effective uncertainty of $\tau$.

To test the SSA method under less ideal signal conditions, the same measurements were repeated after decreasing the pulse energy to $2 \mathrm{pJ}$ (ca. $3 \mathrm{fJ} /$ pixel at maximum), which
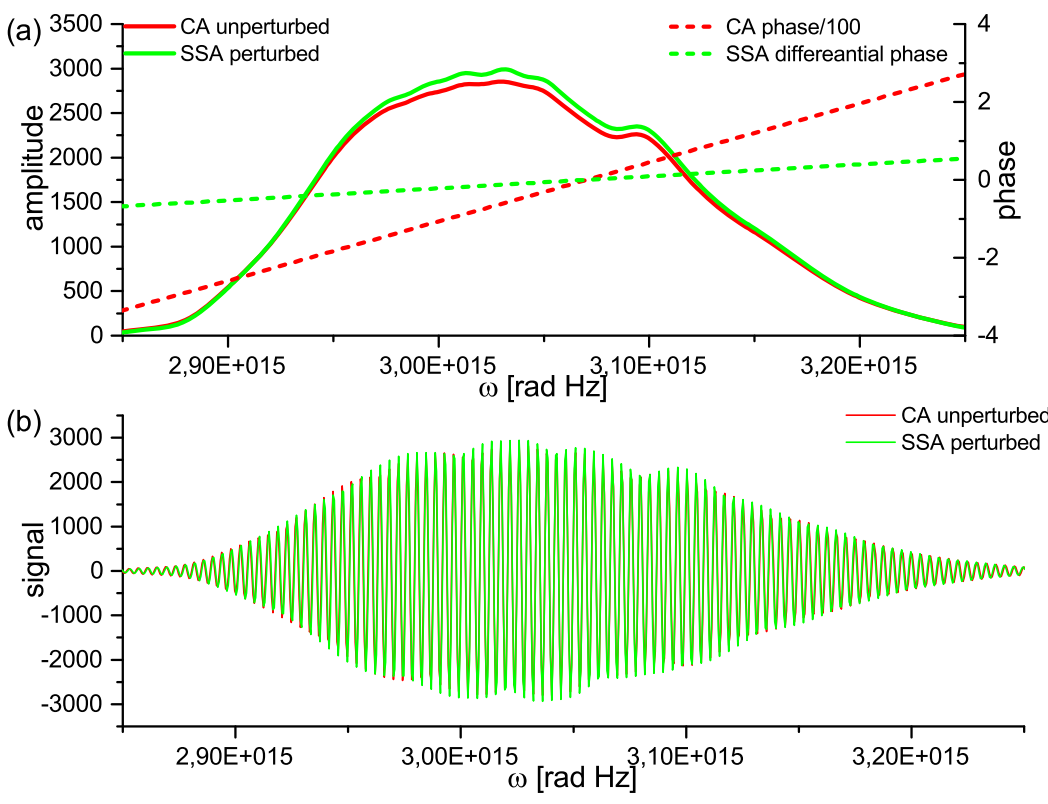

FIG. 3. Comparison between the CA of the unperturbed scan (red line) and the SSA of the perturbed scan (green line) from Fig. 2. SSA on the unperturbed scan is not shown because it is indistinguishable from the respective CA, while the CA of perturbed data is trivially zero. Graphs show (a) amplitude (solid line, left axis) and phase (dashed line, right axis) of the complex field and (b) the reconstructed interferograms, without the DC components. For the sake of clarity, (a) shows the phase of the unperturbed CA (red dashed line, divided by 100) and the difference between the phase of the two fields (green dashed line). 
TABLE I. Chirp parameters in the high power regime by (top to bottom) the conventional analysis (CA) and single shot analysis (SSA) of the same unperturbed dataset, analysis of a single shot interferogram (SSI) from a dataset with perturbation, and SSA on this dataset (SSA pert.). Reported errors are the statistical uncertainty from the fitting procedure and in parentheses $\sigma_{c_{n}}^{S S A}$ from SSA.

\begin{tabular}{llll}
\hline \hline Analysis & \multicolumn{1}{c}{$\tau(\mathrm{fs})$} & \multicolumn{1}{c}{ GVD $\left(\mathrm{fs}^{2}\right)$} & \multicolumn{1}{c}{ TOD $\left(\mathrm{fs}^{3}\right)$} \\
\hline CA & $1518.14 \pm 0.01$ & $-53.0 \pm 0.2$ & $104 \pm 7$ \\
SSA & $1518.12 \pm 0.01(0.12)$ & $-53.1 \pm 0.2(0.8)$ & $108 \pm 7(30)$ \\
SSI & $1518.21 \pm 0.02$ & $-53.4 \pm 0.3$ & $106 \pm 9$ \\
SSA pert. & $1521.22 \pm 0.01(7)$ & $-53.4 \pm 0.2(1)$ & $107 \pm 7(30)$ \\
\hline \hline
\end{tabular}

corresponds to a signal-to-noise-ratio $<1$ (Fig. 5). For the sake of visibility, the spectra were subtracted by the background and zero-frequency-peak, measured by increasing the inter-pulse distance to smear out the interference fringes.

First we validate the SSA in this experimental conditions by comparing the outcome of CA and SSA on the same unperturbed data (first and second rows of Table II). The two analyses give congruent figures (within $3 \sigma$ ), with inter-pulse delays within 1 standard deviation. This result highlights the robustness of SSA to detector noise. Moving to the analysis of the scan with noise, again the CA on the perturbed dataset gives no signal but on the contrary of the previous case, the SSI does not show a clear interference pattern, being dominated by detector noise (Fig. 5). However the SSA can still retrieve the signal both in amplitude and phase (Fig. 6). With respect to the high-power case, we observe more deviations with respect to the reference (red lines). The main discrepancy is observed for $\tau$ which can be explained in analogy to the high power case. The comparison of a single SSI with the SSA of 5000 shots, shown in Fig. 6 and Table II, demonstrates clearly an improvement in the quality of the signal by the SSA. The reconstruction is not as good as for the high intensities and, more relevant, for the CA of low-power unperturbed data. This is somehow foreseeable. Assuming for the sake of simplicity a white detector noise around the dark offset, the CA will average it to zero and then an improvement of the final complex signal is expected. Conversely in the SSA, the SSIs are Fourier transformed before averaging the amplitudes where a white noise will induce a non-zero background. This leads to the loss of spectral contributions to the signal that is much smaller than this noise. Indeed the observed spectral distortion looks like a threshold filter. Noteworthy, these considerations hold also for statistical noise. Not surprisingly, a phase is even more sensitive than an amplitude to white noise: in the tails of the signal, phase contains more and more contributions from the white noise phase, whose spectral density goes as $\omega^{-2}$. Accordingly it reduces the strong dependence of the signal phase, affecting more the lower frequency tail. The behaviour of the SSA differential phase in Fig. 6(a) shows indeed this behaviour. We carried out the SSA increasing progressively the number of spectra from 1 to 5000 and no significant improvement of the reconstruction was observed by averaging more than the first 1000 shots, in agreement with the aforesaid explanation. This shows the limit of the SSA and how it becomes critical for low intensities.

We also observe an increase of the fitting error and of the standard deviations of the parameter distributions especially for the high order phase parameters. Remarkably, now the dominant contribution to noise is the electronic one of the detector, which is a source of noise that does not benefit from an effective fs-ps integration time. This is the reason why uncertainties on SSI parameters and $\sigma_{c_{n}}^{S S A}$ of perturbed and unperturbed ones are substantially comparable within one order of magnitude, on contrast to the case of the high power regime.

To test the capability of the SSA to retrieve higher order phase dispersion and to validate it with ps pulses, a $5 \mathrm{~cm}$ thick fused silica glass (corresponding to a GVD of $5322 \mathrm{fs}^{2}$ and a TOD of $2440 \mathrm{fs}^{3}$ ) was inserted in one of the interferometer

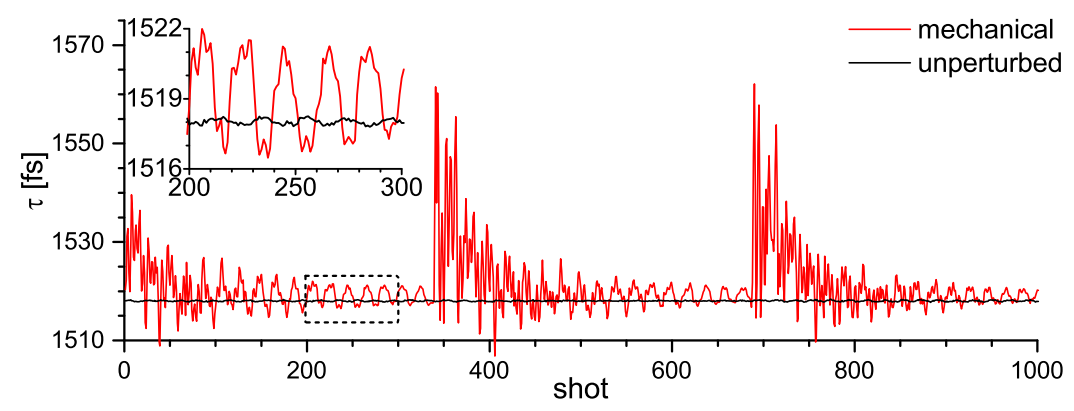

FIG. 4. Shot-by-shot fluctuations of the inter-pulse delay for unperturbed scan (black line) and scans where noise was generated by mechanical fluctuations (red line). The inset shows the 200-300 interval in a magnified scale.

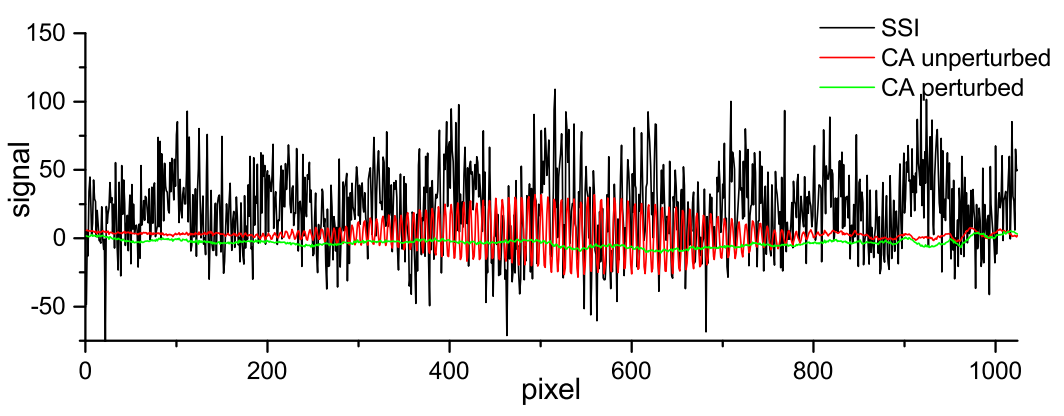

FIG. 5. Measured spectra with a pulse energy of $2 \mathrm{pJ}$ (ca. $3 \mathrm{fJ} /$ pixel at maximum) after subtraction of the background and zero frequency signal. The graph shows the spectrum of a typical SSI (black line) and the CA of a scan with and without perturbation (green and red lines, respectively). 
TABLE II. Chirp parameters in the low power regime, see Table I for notations.

\begin{tabular}{lccc}
\hline \hline Analysis & $\tau(\mathrm{fs})$ & $\mathrm{GVD}\left(\mathrm{fs}^{2}\right)$ & $\mathrm{TOD}\left(\mathrm{fs}^{3}\right)$ \\
\hline CA & $1508.27 \pm 0.05$ & $-54.4 \pm 0.8$ & $80 \pm 50$ \\
SSA & $1508.38 \pm 0.05(4)$ & $-59.0 \pm 0.8(80)$ & $-140 \pm 50(5000)$ \\
SSI & $1517 \pm 1$ & $70 \pm 20$ & $-8000 \pm 100$ \\
SSA pert. & $1523.37 \pm 0.05(11)$ & $-62.7 \pm 0.8(110)$ & $-300 \pm 50(6000)$ \\
\hline \hline
\end{tabular}

arms and the length of the other arm was changed accordingly. The added chirp leads to a broadening of the fringes for shorter frequencies (data not shown) and to an elongation of the pulse to 3 ps.

The phase functions retrieved by the SSA and their chirp parameters (Table III) show again a very good agreement between the perturbed and the unperturbed measurement and the expected values.

Another example of the advantages of single shot detected interferometry is the possibility to measure the phase stability of a setup, to identify sources of noise and to monitor drifts by evaluating $\tau$ for each SSI and analysing how it changes during the scan.

This is shown in Fig. 7 where fluctuations of $\tau(\delta)$ caused by tapping and by blowing pressurized $\mathrm{CO}_{2}$ are compared with an unperturbed scan. The three traces look completely different and even a simple visual inspection allows us to identify which trace is affected by mechanical instabilities or air turbulences. As commented before, the former shows a typical damped vibrational pattern and it can be seen clearly when the setup was perturbed. The latter shows increased noise with slow wavy changes without a vibrational structure.

This plot allows also us to identify sudden changes in the optical path (e.g., due to a mount released during the scan) and slow drift. For example, a 5 fs shift can be clearly identified in the unperturbed data set around shot 2900, while slow drifts are easily tracked down in both the black and the red curves after
TABLE III. Chirp parameters with a $5 \mathrm{~cm}$ thick fused silica glass in one arm (see Table I for notations). For the unperturbed scan, only the first 2750 shots of the scan were evaluated due to a drift that occurred during the scan (Fig. 7).

\begin{tabular}{llll}
\hline \hline Analysis & \multicolumn{1}{c}{$\tau(\mathrm{fs})$} & \multicolumn{1}{c}{ GVD $\left(\mathrm{fs}^{2}\right)$} & \multicolumn{1}{c}{ TOD $\left(\mathrm{fs}^{3}\right)$} \\
\hline CA & $1240.2 \pm 0.1$ & $4925 \pm 2$ & $2000 \pm 80$ \\
SSA & $1240.3 \pm 0.1(0.3)$ & $4925 \pm 2(5)$ & $2000 \pm 80(300)$ \\
SSI & $1240.3 \pm 0.1$ & $4922 \pm 3$ & $1930 \pm 120$ \\
SSA pert. & $1253.6 \pm 0.1(11)$ & $4925 \pm 2(0)$ & $2000 \pm 80(1000)$ \\
\hline \hline
\end{tabular}

shots 3000 and 2000, respectively. The single shot detection allows in such a case to identify the problem and to still analyze the data either by performing the CA for the stable part of the measurement or by using the SSA to compensate for the drift.

Concerning the other sources of noise not benefitting from an effective fs-ps integration time, as electronic detection noise or computational errors, the comparison of SSI uncertainty with $\sigma_{c_{n}}^{S S A}$ can be an excellent criterion to identify such kinds of sources and evaluate their weight on the overall phase noise.

It is worth mentioning that the herein proposed approach cannot compensate for spatial phase noise through the beam transverse mode: shot-by-shot phase differences at the same wavelengths but at different positions through the beam will be mixed up together at the detector plane, degrading the SSI. In this respect, we can say that single-shot detection can compensate for the average fluctuation of the phase front, pushing the problem to the next higher order: instantaneous spatial distortions of the phase front.

In the case of light sources inherently with phase front noise, a possible solution is to set up a spatial filter to select only the pure transversal Gaussian mode. This is done at the cost of pulse intensity but will allow adopting the approach herein presented. Spatial phase noise and distortion can also be induced by density differences of air, vibrations of mirror surfaces, and surface imperfections. It is difficult to give (a)

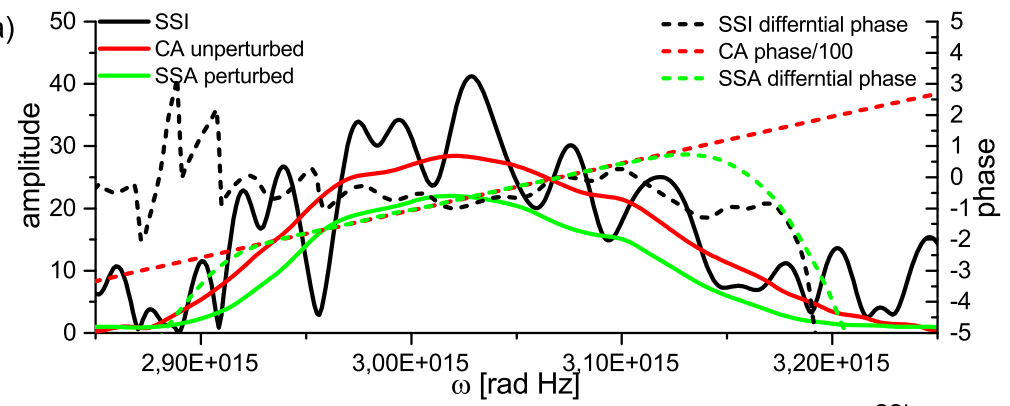

(b)

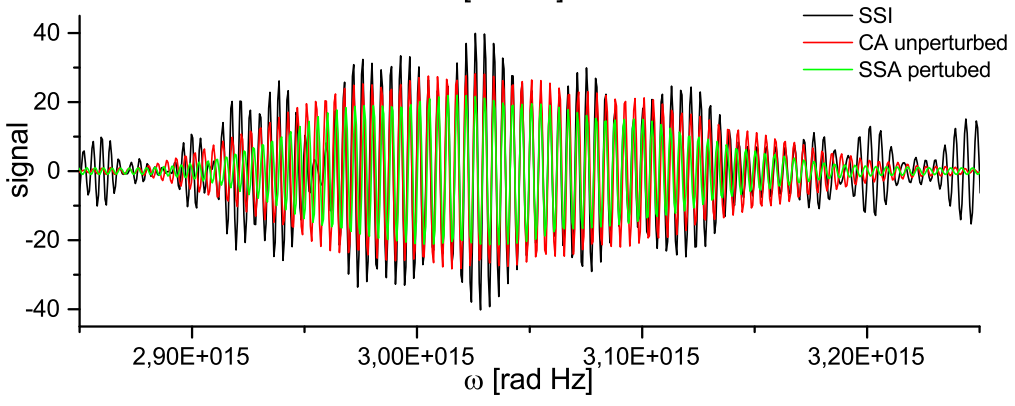

FIG. 6. Comparison among the SSI, the CA of the unperturbed scan, and the SSA of the perturbed scan from Fig. 5 (black, red, and green lines, respectively). The resulting complex field (a) and the reconstructed interferograms (b) are plotted. Solid (dashed) lines show the magnitude (phase) of the signal. For the sake of clarity, the phase of the unperturbed measurement is divided by 100 while only the difference between the phase of the SSI and of the SSA with respect to the CA is reported. 


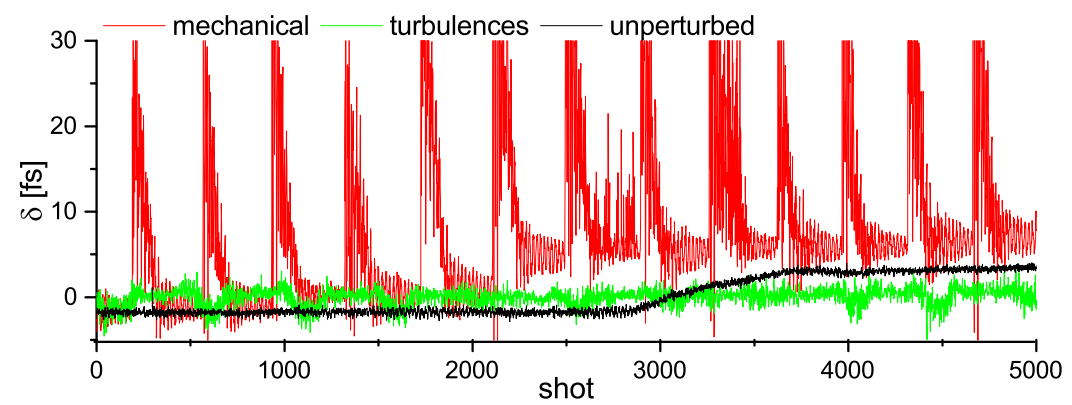

FIG. 7. Shot-by-shot fluctuations of the inter-pulse delay $(\delta)$ for unperturbed scan (black line) and scans where noise was generated by mechanical fluctuations (red line) or by air turbulences (green line) with a sudden shit occurring around shot 2850 in the unperturbed scan. a typical value for such terms because they strongly depended on the experimental conditions (pressure and humidity, beam size, temperature, vibration and heat isolation, propagation length through air, etc.) but, for instance, amplitudes of surface vibrations at room temperature can be $0.1-0.05 \AA$ depending on the substrate, ${ }^{21,22}$ with a square-root dependence on the substrate temperature (at liquid nitrogen temperatures, a value as low as 0.02 can be reached ${ }^{22}$ ). Then according to the criterion $\Delta \lambda=\frac{\lambda}{100}$, the validity limit of our approach would be set between 10 and $1 \AA$ (1-10 keV), with a suitable choice of mirror substrates and temperature control. The other processes (air density inhomogeneity and surface quality) are more difficult to estimate but they can be easier controlled with suitable strategies (e.g., having most of the propagation in vacuum and imaging on the mirror surfaces). They can be removed inserting spatial filters close the sample. Conversely, since a spatial filter also consists of mirrors, it cannot efficiently suppress the effect of surface vibrations.

\section{CONCLUSION}

In this article, we prove the capability of broadband singleshot detection of fs and ps pulses to perform Fourier transform spectral interferometry with non-phase-stable setups. Ultimately it is based on the acquired capability to average directly complex fields rather than only power spectra.

This approach can have a terrific impact on the implementation of optical phase sensitive techniques, significantly simplifying their implementation and allowing the conception of more sophisticated schemes. In particular, we anticipated in the Introduction that it is especially relevant to FT-FWM. This is because it is also able to measure with high precision the instantaneous delay between two pulses (see Table I and relative discussion). In a FT-FWM experiment, it is indeed necessary a heterodyne detection of the signal but also a precise knowledge of the delay between the excitation pulses $\left(\tau_{c o h}\right)$. A beamsampler just before the sample could be used to generate a replica of the latter beams in order to measure their interpulse delay by the SSI. A synchronized multi-camera detection system (as the one we developed) allows us to measure simultaneously and without time ambiguity the interferogram of the heterodyne signal and $\tau_{c o h}$, which is all it is required to carry out a FT-FWM experiment. SSIs will be grouped according to $\tau_{c o h}$ and analyzed with the SSA herein proved.

This scheme based on generating a replica of all the beams just before the sample and using single-shot detection do measure simultaneously the heterodyne signal and all the relevant interpulse delays has several advantages, when compared with other schemes for 2D electronic spectroscopy. ${ }^{6}$ So far UV and visible FT-FWM spectrographs have been implemented by conceiving a design to preserve passively the phase stability ${ }^{8-10,23}$ or based on the pulse-shaping technology. ${ }^{3,12-14}$ This makes the extension of such schemes to shorter wavelengths or higher orders of wave mixing hard or even impossible. Conversely the innovation of our approach concerns essentially how signals are acquired and analysed, so it does not need any special detection geometry or optical design. This allows the realization of relatively easy and inexpensive coherent wave-mixing setups (four or even higher orders) with conventional optics and without a phase-stable design. This and the fulfilment of the phase stability criterion for virtually any wavelength make finally possible to cover with such coherent techniques spectral regions from UV to hard X-rays.

Actually this is already possible with the cameras developed for this work by extending their detection range to $\lambda<200 \mathrm{~nm}$ with scintillators or down-converting phosphors, provided that fs to $\mathrm{ps}$ sources with a repetition rate as high as $8 \mathrm{kHz}$ are used (or up to $500 \mathrm{kHz}$ but with an acquisition duty cycle equal to the ratio between the detection and laser repetition rates) and the signal-to-noise ratio of a single-shot detected interferogram is high enough (see Fig. 6 and relative discussion).

This proof of principle has also implications important for the field of diagnostics based on interferometry as pulse characterization, high precision length measurements, identification and monitoring of noise sources (physical instabilities, electronics and detection, and computational data treatment), etc.

\section{ACKNOWLEDGMENTS}

The authors acknowledge the support by the European Union (ERC Starting Grant No. 279599-FunctionalDyna), the Swiss NSF through the NCCR MUST and project n. 200021_172696, and the Swiss State Secretariat for Education Research and Innovation through the European programme COST (Action CM1202). They also gratefully thank Professor Thomas Feurer and Dr. Egmont Rohwer for help and discussions.

\footnotetext{
${ }^{1}$ S. Mukamel, Principles of Nonlinear Optical Spectroscopy (Oxford University Press, 1999).

${ }^{2}$ D. M. Jonas, Annu. Rev. Phys. Chem. 54, 425 (2003).

${ }^{3}$ R. J. D. Miller, A. Paarmann, and V. I. Prokhorenko, Acc. Chem. Res. 42, 1442 (2009).
} 
${ }^{4}$ B. A. West, B. P. Molesky, P. G. Giokas, and A. M. Moran, Chem. Phys. 423, 92 (2013).

${ }^{5}$ S. M. Gallagher, A. W. Albrecht, T. D. Hybl, B. L. Landin, B. Rajaram, and D. M. Jonas, J. Opt. Soc. Am. B 15, 2338 (1998).

${ }^{6}$ F. D. Fuller and J. P. Ogilvie, Annu. Rev. Phys. Chem. 66, 667 (2015).

${ }^{7}$ V. Volkov, R. Schanz, and P. Hamm, Opt. Lett. 30, 2010 (2005).

${ }^{8}$ M. L. Cowan, J. P. Ogilvie, and R. J. D. Miller, Chem. Phys. Lett. 386, 184 (2004).

${ }^{9}$ T. Brixner, T. Mančal, I. V. Stiopkin, and G. R. Fleming, J. Chem. Phys. 121, 4221 (2004).

${ }^{10}$ U. Selig, F. Langhojer, F. Dimler, T. Löhrig, C. Schwarz, B. Gieseking, and T. Brixner, Opt. Lett. 33, 2851 (2008).

${ }^{11}$ S.-H. Shim and M. T. Zanni, Phys. Chem. Chem. Phys. 11, 748 (2009).

${ }^{12}$ N. Krebs, I. Pugliesi, J. Hauer, and E. Riedle, New J. Phys. 15, 085016 (2013).

${ }^{13}$ T. Hornung, J. C. Vaughan, T. Feurer, and K. A. Nelson, Opt. Lett. 29, 2052 (2004).
${ }^{14}$ Y. Rodriguez, F. Frei, A. Cannizzo, and T. Feurer, J. Chem. Phys. 142, 212451 (2015).

${ }^{15}$ A. Cannizzo, Phys. Chem. Chem. Phys. 14, 11205 (2012).

${ }^{16}$ F. Bencivenga, R. Cucini, F. Capotondi, A. Battistoni, R. Mincigrucci, E. Giangrisostomi, A. Gessini, M. Manfredda, I. P. Nikolov, E. Pedersoli, E. Principi, C. Svetina, P. Parisse, F. Casolari, M. B. Danailov, M. Kiskinova, and C. Masciovecchio, Nature 520, 205 (2015).

${ }^{17}$ P. Baum, S. Lochbrunner, and E. Riedle, Opt. Lett. 29, 1686 (2004).

${ }^{18}$ P. Baum, S. Lochbrunner, and E. Riedle, Appl. Phys. B 79, 1027 (2004).

${ }^{19}$ L. Gallmann, C. Cirelli, and U. Keller, Annu. Rev. Phys. Chem. 63, 447 (2012).

${ }^{20}$ L. Lepetit, G. Chériaux, and M. Joffre, J. Opt. Soc. Am. B 12, 2467 (1995).

${ }^{21}$ W. Steurer, A. Apfolter, M. Koch, W. E. Ernst, E. Sndergrd, J. R. Manson, and B. Holst, Phys. Rev. B 78, 045427 (2008).

${ }^{22}$ J. S. Becker, R. D. Brown, E. Johansson, N. S. Lewis, and S. J. Sibener, J. Chem. Phys. 133, 104705 (2010).

${ }^{23}$ T. A. Gellen, L. A. Bizimana, W. P. Carbery, I. Breen, and D. B. Turner, J. Chem. Phys. 145, 064201 (2016). 